\title{
SOBRE EL SENTIDO MORAL DE LA LIBERTAD
}

\author{
Jacob Buganza \\ Universidad Veracruzana
}

Resumen: En este trabajo buscamos exponer en qué sentido es totalmente plausible hablar del libre arbitrio de la voluntad en filosofía, contrario a lo que afirma Luis E. Hoyos. En primer lugar, revisamos el concepto de voluntad para ver cómo de su propio ser brota el libre arbitrio (pues no todo acto de la voluntad es libre, como se ve en el artículo). Pero también el libre arbitrio de la voluntad es limitado, y siguiendo a Rosmini indicamos algunos de estos límites.

Palabras clave: Voluntad, libre arbitrio, moralidad, límites de la libertad y rosminianismo.

On the moral sense of freedom

Abstract: In this paper we seek to explain in what sense is entirely plausible talk of free will of the will in philosophy, contrary to the assertions of Luis E. Hoyos. First of all, we review the concept of will to see how their own being the free will springs (not every act of the will is free, as seen in the article). But free will of the will is limited, and following Rosmini we indicate some of these limits.

Keywords: Will, free Will, morality, limits of freedom and rosminianism..

Recibido: 29/3/2016 Aprobado: 21/4/2017

\section{Introducción}

En este trabajo sostenemos que el sentido de la libertad, o de la libertad de la voluntad, consiste en reconocer a los entes por aquello que son, que es lo mismo que decir que el sentido de la libertad es la moralidad. En primer lugar dilucidaremos la noción de voluntad, para luego discutir sobre el senti- 
do que tiene la libre voluntad y la diferencia que guarda con la libertad de acción. Sostenemos que hay vínculo entre ambas formas de visualizar la libertad, pero resulta más fundamental la primera, pues la libertad consiste en la elección del juicio a partir del cual se actúa. Finalmente enlistaremos algunos límites de la libertad, pues es evidente que no es absoluta, sino siempre circunscrita.

\section{Sobre el concepto de voluntad}

En primer lugar, conviene contraponer el instinto y la voluntad, pues con frecuencia se suelen atribuir elementos de uno a otra y viceversa. Es verdad que tanto el instinto como la voluntad convergen en ser potencias activas de la actividad vital del hombre, mientras que las funciones del sentir y el conocer podrían considerarse pasivas, aunque estrictamente el conocer sea receptivo más que activo, si tiene razón Rosmini al decir "un ente puede recibir en sí a otro sin confundirse con él, como por ejemplo los objetos conocidos están en el alma de quien los conoce, y esta potencialidad da lugar a una clase que llamaremos potencias receptivas. A veces un ente, recibiendo la acción de otro, remane en cierta forma modificado, y esta pasividad suya da lugar a otra cosa, la de las potencias pasivas. Finalmente, el ente mismo puede efectuar actos que son accidentales, y así se le atribuye la relación que llamamos potencias activas" ${ }^{\text {. }}$.

${ }^{1}$ Antonio Rosmini, Psicologia (Roma, Cittá Nuova, 1988), n. 860. 
La voluntad, a diferencia del instinto, que la tradición ha llamado en general el apetito sensitivo, es una potencia activa iluminada por el intelecto, por lo cual no actúa sin conocimiento intelectual. El instinto, ciertamente, actúa de acuerdo con las condiciones psico-físicas del agente y que inician con un sentimiento; pero la voluntad es la inteligencia que obra, y por ello es llamada appetitus intellectivus. Por ello es que:

\footnotetext{
...actus voluntatis nibil aliud est quam inclinatio quaedam consequens formam intellectam, sicut appetitus naturalis est inclinatio consequens formam naturalem. Inclinatio autem cujuslibert rei est in ipsa re per modum ejus. Unde inclinatio naturalis est naturaliter in re naturali; et inclinatio quae est appetitus sensibilis, est sensibiliter in sentiente; et similiter inclinatio intelligibilis, quae est actus voluntatis, est intelligibiliter in intelligente, sicut in primo principio, et in proprio subjecto"2.
}

Así pues, el acto de la voluntad, que es a través del cual se conoce la naturaleza de esta potencia, es el acto de querer que sigue al conocimiento intelectual. Por tanto, no se refiere a cualquier apetito, sino sólo al apetito que sigue al conocimiento intelectual, como suele confundirse. En efecto, el acto voluntario es una especie de actos humanos, pues estos últimos son aquéllos que efectúa el hombre a diferencia de otros entes naturales. Ciertamente el hombre, a diferencia de los animales en general, lleva a cabo actos distintos, como son los intelectuales y los voluntarios. Si la voluntad es la potencia por la cual tiende al bien conocido, entonces es evidente que los

${ }^{2}$ Thomae Aquinatis, Summa theologiae, I, q. 87, a. 4c. 
actos voluntarios corresponden a las apetencias que siguen al conocimiento intelectual.

El propio Descartes llega a decir que "lintellection est proprement la passion de l'âme, et l'acte de la volonté son action", como bien recuerda Jean-Luc Marion, y de donde explica que "ahí donde conozco, soy pasivo. Pero el conocimiento da un objeto que puede inmediatamente ser querido (être vou$l u$ ), esto es, aprobado o rechazado. Se sigue que la distinción entre la voluntad y la intelección es una distinción débil (faible), por lo que son dos modalidades de la misma cognición. ¿Qué quiere decirse? Respuesta: que cuando conozco algo, inmediatamente soy llamado a quererla positiva o negativamente" 3 .

Marion acierta al considerar la voluntad como la llamada a querer algo positiva o negativamente. En efecto, la voluntad, en especial la libre voluntad, por definición, no está sujeta a la violencia ${ }^{4}$. Pero Marion, al menos siguiendo las hormas de Descartes, no llega a ver que los actos de la voluntad son al menos de dos tipos, a saber, voliciones afectivas y voliciones apreciativas; tampoco todo acto de la voluntad es libre, como veremos más adelante, y por ello resulta necesario conceptuar al liberum arbitrium para diferenciarlo

${ }^{3}$ Jean-Luc Marion, Cours sur la volonté (Louvain, Presses Universitaires de Louvain, 2014), p. 82.

${ }^{4}$ Ya Aristóteles señala que la violencia significa un principio que viene de fuera, de tal manera

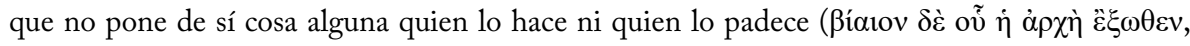

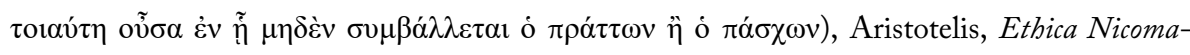
chea, III, 1, 1110a2-3. 
de cualquier otro acto voluntario, lo cual, a nuestro juicio, Luis Eduardo Hoyos no tiene en consideración, como veremos en el aparte siguiente.

En efecto, el acto que llamamos volición afectiva de la voluntad es un acto espontáneo de la inteligencia, como sucede siempre en el caso de los niños, y de manera frecuente en los adultos, que no requieren conceptuar todo lo que conocen en términos "medio" y "fin" para querer racionalmente algo que conocen. Mediante la volición afectiva se percibe intelectivamente un objeto, y se le apetece racionalmente, y no más. En cuanto lo conocido o percibido intelectivamente es considerado grato al sentido, se tiende a él. Pero no hay todavía en estas voliciones afectivas un "juicio" sobre el valor de la cosa apetecida, en cuanto el juicio exige una regla abstracta, que el hombre en todo momento no tiene presente. A la potencia de la percepción intelectiva $\mathrm{Co}^{-}$ rresponde de manera inmediata una espontánea volición afectiva. De hecho, puede decirse que por su vínculo a la sensibilidad la volición afectiva está íntimamente conectada con el instinto, pero ya no es mero instinto.

Pero una vez que el hombre hace uso de la abstracción y la reflexión, halla reglas precisamente abstractas para juzgar aquello que conoce. La abstracción supone a la reflexión, porque presupone que se ponga atención en la percepción y las ideas, restringiéndose a observar algo en común o en alguna propiedad particular. De las percepciones se obtienen las ideas específicas, y de éstas las ideas genéricas, las cuales pueden serlo siempre más y más, hasta las más universales y abstractas. Por ello es que la abstracción es una función 
de la reflexión y de la atención, y se desarrolla en muchísimos grados ${ }^{5}$. Sólo cuando se tienen las reglas abstractas, que son ciertamente ideas ${ }^{6}$, entonces puede darse el acto de la volición apreciativa.

Cuando un objeto es apreciado por nosotros como bueno, entonces se puede apetecer y querer no ya sólo mediante el instinto animal, sino por un instinto intelectual que nace como consecuencia de este juicio, instinto que es completado y confirmado por un decreto explícito de la voluntad. Por tanto, la volición apreciativa se compone de tres actos, a saber, el juicio apreciativo, el instinto intelectual que inclina al hombre hacia el bien que ha apreciado, $y$ el decreto de la voluntad que establece si quiere dejar satisfecho este instinto o no.

Sólo una vez que se da este estadio de desarrollo en el hombre, es que puede comenzar a hablarse de la elección, pero en el acto apreciativo todavía no se ha dado el acto electivo y tampoco el acto libre. En efecto, sólo cuando se ha alcanzado el momento en que se dan voliciones apreciativas puede darse cita la elección y la libertad; la primera consiste precisamente el preferir alguno de los bienes que están a disposición o que es posible obtener; la libertad se da cuando se pueden elegir bienes sólo subjetivamente considerados o bienes objetivos. Ahora bien, no tiene sentido la elección ni la libertad si se refiere ésta a lo que es imposible; sólo en el marco de lo posible es que el acto electivo tiene sentido. Pero antes de discutir con mayor profundidad la natu-

${ }^{5}$ Antonio Rosmini, Antropologia in servizio della scienza morale (Roma, Citta Nuova, 1981), nn. 541-542.

${ }^{6}$ Cf. Antonio Rosmini, Principi della scienza morale (Roma, Città Nuova, 1990), 505pp. 
raleza del acto electivo y de la libertad, retomemos una parte de la discusión contemporánea en torno a la libertad de la voluntad.

\section{Libertad de la voluntad y libertad de acción}

En un ensayo muy interesante, Luis Eduardo Hoyos llama la atención sobre el concepto de "libertad de la voluntad" (es evidente que no todo acto de la voluntad es libre, pues hemos hablado ya de las voliciones afectivas, que siguen espontáneamente al conocimiento intelectual; también hemos hablado de las voliciones apreciativas, a partir de las cuales tienen origen los actos libres, pero no son todavía equivalentes). Partiendo de la distinción entre "libertad de acción" y "libertad de la voluntad", confiesa Hoyos que no ve "cómo pueda articularse un discurso sobre la libertad de la voluntad sin tener en cuenta la de la acción, o sin tener en cuenta que el querer, los deseos, las preferencias, etc., tienen ante todo significado en un contexto relativizado, o performativo"7.

La tesis de Hoyos pretende contraponerse la de otros autores, entre los que cita a Frankfurt y Tugendhat, para quienes el tema de la libertad humana consiste o se resuelve esencialmente en el tema de la libertad de la voluntad. Para penetrar en este asunto, dice que acepta prima facie que la libertad de querer puede entenderse en relación con la acción y, así, con la libertad de acción, o sin ella. Se pregunta en qué consiste la libertad de la voluntad sin la de acción, y retoma la definición de Beckermann que dice que

\footnotetext{
${ }^{7}$ Luis Eduardo Hoyos, “El sentido de la libertad”, Ideas y valores, n. 141, Bogotá, 2009, p. 91.
} 
"una persona es libre en su querer cuando tiene la facultad de determinar su voluntad, de determinar qué motivos, deseos y convicciones han de ser eficientes para actuar", y de inmediato agrega Hoyos: "Lo importante de esta definición, repito, está en que comprende imbricadas a la libertad de la voluntad y a la de actuar. De modo que, para que podamos llevar a cabo el ejercicio que propongo, tendríamos que suprimir la última parte de la definición, es decir, considerar la libertad de la voluntad como la capacidad o característica de una persona de determinar su querer (sus motivos, sus deseos, etc.), sin entrar a considerar el contexto performativo de esos deseos. Sostengo que esa noción es vacía, si no ininteligible. Y, sin embargo, parece haber merecido un lugar en la historia de la filosofía"

No vamos a defender la definición de Beckermann en este momento, pero sí a criticar la postura de Hoyos. Este último no llega a distinguir entre las ideas de querer y actuar. Es verdad que están íntimamente relacionadas una con la otra, pero no son equivalentes. Es un hecho que el propio lenguaje pone de manifiesto de manera frecuente: se hace lo que no se quería. Hoyos quiere reducir el querer a la acción, pues le parece ininteligible que haya una diferencia entre los dos actos. Pero tan son distintos que los llamamos con distintos nombres. Además, la definición de Beckermann no es ininteligible; al contrario, es bastante clara en su formulación. En efecto, la libertad consiste en la capacidad que tiene el agente para determinar su voluntad; y esto se explica porque el agente es capaz de determinar con base en qué motivos se determina. No hay, a nuestro juicio, ninguna ininteligibilidad en la defini-

${ }^{8}$ Ibid., p. 92. 
ción de Beckermann. De hecho, hace muy bien en partir de la voluntad para dar cuenta de la libertad; no se puede partir de la libertad sin más para explicarla. Se debe recurrir a la potencia o facultad que es precisamente libre para determinarse, y esto es lo mismo que decir que determina su querer con base en motivos (preferentemente en razones y no en meros impulsos, pues los motivos son género y las razones y los impulsos especie); es decir, la libertad de la voluntad consiste en determinar el querer por ciertos motivos y en no querer otra cosa (lo cual no quiere decir que no se le quiera en términos absolutos) por otros motivos.

Lo que pretende Hoyos derivar de esta discusión es que la distinción entre la libertad de la voluntad y la libertad de la acción ha llevado al postulado de la "idea" de un liberum arbitrium indiferentiae. De ahí asienta este filósofo: "Pienso que la idea de la libertad de la voluntad, independientemente de la libertad de acción, o de la libertad para actuar, no es una idea interesante filosóficamente, pues es la principal promotora de la ficción metafísica de una libertad incondicionada. Si el de libertad, y el de libertad de la voluntad, es un concepto capturable, prometedor, interesante, debemos abandonar la idea de que pueda haber algo así como una libertad incondicionada. Hay condiciones de la libertad. Buena parte de la tarea de pensar la libertad, si no la principal parte, consiste en pesar esas condiciones"

${ }^{9}$ Ibidem. Es claro que afirmar que el problema del libre arbitrio sea un problema de índole metafísico, pero en un sentido peyorativo, se debe a la escuela inglesa, Cf. Mario De Caro, Il libero arbitrio. Una introduzione (Roma y Bari, Laterza, 2009, 3a. ed), 192pp. Además, parece que Hoyos no tiene en cuenta que liberum arbitrium significa, con propiedad, "juicio no nece- 
No se ve con claridad en el texto de Hoyos quién propone una libertad absoluta, que es lo mismo para él que incondicionada. En general, parece que los filósofos proponen una noción de libertad condicionada; qué tan condicionada esté, depende de los límites que se agreguen ${ }^{10}$. Si se refiere a Descartes, Hoyos parece confundir la esencia o idea de la libertad con la libertad real, es decir, con la libre voluntad que cada individuo humano posee. Cada individuo está condicionado por múltiples circunstancias, que van desde las condiciones biológicas hasta su historia personal ${ }^{11}$. En esencia, la libertad consiste en no estar coaccionado o violentado, y en este sentido se puede hablar de la esencia de la libertad; otra cosa es la libertad de cada agente real. Estos planos parecen confundirse en Hoyos. Y tan se confunden que estima que no es interesante reflexionar sobre la libertad, por el simple

sario", pues arbitrium se traduce por "juicio" sin dificultad y liberum por "no necesario". Así que ni siquiera en su acepción negativa de "metafísico" pueda entenderse la afirmación de Hoyos.

${ }^{10}$ Estamos de acuerdo con Sciacca cuando escribe que "Voluntad libre no significa incondicionada — voluntad imposible, una abstracción-, en cuanto no puede sustituirse a las otras fuerzas que mueven al hombre, ni a los entes hacia los cuales es movida. Su objetivo, no el de comprimir o extirpar tendencias e instintos, sino de regular sus empeños, aceptaros o modificarlos, orientarlos [...] La libertad comienza por el acto con el cual cada hombre asume la propia naturaleza como un elemento de la persona en su integralidad, para hacerse la persona que es con esa naturaleza [...] Su naturaleza dada deja de ser un dato y se vuelve responsabilidad personal', Michele Federico Sciacca, La libertà e il tempo (Milano, Marzorati, 1965), pp. 23-24.

${ }^{11}$ Cf. Jacob Buganza, Filosofía de la mente, voṽ y libertad (Xalapa, Universidad Veracruzana, 2014), 164pp. 
argumento de que no le resulta interesante "filosóficamente", como sí lo ha sido para cientos de filósofos, entre ellos Schopenhauer ${ }^{12}$.

La distinción entre la idea de la libertad y la libertad de cada agente particular parece entreverse sólo en el texto de Hoyos, pues afirma: "La libertad de querer es libertad de elegir, y no es posible concebir la idea de una elección que no esté referida a un número determinado de cosas por elegir y a la capacidad de realizar la elección. Nadie puede elegir en el vacío, es decir, si no tiene qué elegir y si no tiene cómo elegirlo. Y no es cierto, por otra parte, que se pueda elegir infinitamente. Una condición mínima de la posibilidad de elegir es que el número de opciones sea finito"13. Si se preguntara a qué cosa tiende la libertad de la voluntad considerada como idea, sólo se podría responder que a un bien, pero el ser y el bien son lo mismo, sólo que vistos desde aspectos diversos (ens et bonum convertuntur); luego, la voluntad tiende al ser ${ }^{14}$. Y si el ser, al menos como idea, es infinito, entonces la voluntad tiende al infinito. Pero esto se refiere, hay que repetirlo, a la idea de la libre

12 Tan importante de hecho ha sido el tema de la voluntad para Schopenhauer, que la eleva a principio absoluto de todo lo real. Cf. Arthur, Schopenhauer, El mundo como voluntad y representación, L. IV. De hecho, para la filosofía la libertad de indiferencia no es más que una hipótesis: En la elección entre dos bienes particulares incompatibles, la voluntad los tiene en cuenta, considerando el grado de bondad que tienen, de lo atractivos que son, o bien actúa sin tenerlos en cuenta, prescindiendo de ellos. En este segundo caso se tiende al vacío, y no tanto al objeto, al bien; se trataría de un acto voluntario sin causa, sin motivo, sin razón. La libertad de indiferencia sería equivalente a una voluntad que sería indiferente justamente a los bienes, Cf. Giuseppe Morando, Il problema del libero arbitrio (Milano, Cogliati, 1895), pp. 17-18.

${ }^{13}$ Luis Eduardo Hoyos, art. cit., p. 92.

${ }^{14}$ Cf. Jacob Buganza, El ser y el bien (Stresa, Edizioni Rosminiane Sodalitas, 2010), 116pp. 
voluntad. Mas cuando nos referimos a una libre voluntad particular, es evidente que las opciones son finitas porque finito es el agente libre. Por ello, nuevamente a nuestro juicio, Hoyos confunde dos cosas: la idea de la libre voluntad y la voluntad de un agente particular.

Es verdad que Hoyos lo que busca es sostener que la libertad de la voluntad no puede separarse de la libertad de la acción. No nos parece que haya error en esta propuesta; simplemente lo que buscamos subrayar es que se trata de dos cosas distintas, esto es, que una cosa es la libertad de querer y otra la libertad para actuar, la cual frecuentemente se asocia a la obra externa. Puedo querer una cierta cosa y, sin embargo, es posible que no pueda realizarla, por diversidad de condiciones. Hoyos tiene presente con mucha justicia la normatividad que, gracias a la educación y a otras circunstancias, se encuentra presente en el sujeto humano. Se trata, ciertamente, de una potencia virtual del hombre, en cuanto requiere no sólo de educación, sino que presupone un sujeto educable, es decir, que haya desarrollado las funciones o popotencias resultantes de la razón, como es ciertamente la reflexión. Lo que queremos subrayar simplemente es que una cosa es la idea de la voluntad, o de la libre voluntad, que consiste en el primer acto por el cual se crea una potencia humana, es decir, cuando se une ésta a su término (la voluntad tiende al bien conocido); y otra cosa son los actos segundos, que consisten en la multiplicidad de determinaciones que adquiere el acto primero. Lo permanente del acto libre voluntario es que se ejercita hacia un bien conocido que se prefiere por encima de otros; lo que varía o la multiplicad se da merced a los diversos objetos que son susceptibles de ser elegidos. 
Sobre el sentido moral de la libertad

\section{Sobre el sentido moral de la libertad}

Cabe ahora dilucidar en qué sentido se habla de "acto libre". Es frecuente confundir entre el acto voluntario, el acto electivo y el acto libre. Pero esencialmente son distintos, y Rosmini brinda una clave para diferenciarlos. En efecto, la voluntad puede querer sin elegir, que es lo que sucede con las voliciones afectivas; por tanto, elegir (scegliere) no es un carácter esencial del acto de la voluntad. El acto de la voluntad simplemente consiste en tender al bien; a qué bien se tienda es precisamente lo indeterminado de la voluntad:

Voluntas est quidem secundum ordidem naturae determinata ad unum commune quid est bonum, sed indeterminate se habet respectu particularium bonorum ${ }^{15}$.

Ahora bien, tampoco en las voliciones apreciativas se da necesariamente elección. Sólo hay elección, como hemos dicho, cuando el agente tiene de frente dos o más objetos, de entre los cuales prefiere unos y posterga otros, debido a que no es posible, si es el caso, obtenerlos todos. Por ello es evidente que sólo cuando hay colisión entre los bienes posibles es que el acto electivo se da cita. ¿Entre qué bienes puede darse esta colisión? La observación suministra la respuesta: entre bienes sensitivos, entre bienes sensitivos y bienes inteligibles pero subjetivos; $y$ entre bienes subjetivos y el orden objetivo. Entre los bienes de orden sensitivo, se prefiere el más placentero, y en este caso la volición necesariamente elige, por decirlo así, a dicho bien.

${ }^{15}$ Thomae Aquinatis, Summa theologiae, I-II, q. 13, a. 2c. 
Cuando colisionan los bienes sensitivo e inteligible, ya se da propiamente elección, como cuando se elige la salud en vez del placer inmediato y momentáneo de la comida que no nutre.

Pero donde la libertad se da con todo su calado es cuando se debe elegir entre el bien subjetivo y el bien objetivo. El hombre es capaz de elegir entre subyugar lo subjetivo a lo objetivo, o en no hacerlo. El hombre "está llamada a volver omnipotente lo invisible, lo ideal, la verdad, la justicia, sobre todo lo que es visible, sobre todo lo real, sobre el universo y todo aquello que hay en él, todo lo bello, grande, seductor y encantador" ${ }^{16}$. Por ello es que Sciacca, a nuestro juicio, acierta en decir que la voluntad, al nivel de la libertad de elección, esto es, que la libre voluntad, es el reconocimiento de todo entre en la "indiferencia" por cualquier interés vital y mundano; sólo se mueve por el amor y, por ello, no es movido por el deseo de posesión o de uso. Consiste en amar las cosas simplemente porque son entes. En este sentido la elección es por sí misma liberadora: "Ama a todo ente con todo el amor que se le debe -el que corresponde a su ser y por ello siempre es amor total- pero no a todos con el mismo amor y ninguno con amor absoluto: reconocimiento objetivo del ente elegido pero sin quitar nada a ningún otro, que siempre permanece como un elegible: la predilección por uno —un bien que es mi bien - incluye también la de los otros — todo el orden del ser- en cuanto en la elección motivada por el amor por el ente en cuanto ser, todos los entes son reconocidos como merecedores de predilección" ${ }^{17}$.

${ }^{16}$ Antonio Rosmini, Antropologia in servizio della scienza morale, Op. cit., n. 582.

${ }^{17}$ Michele Federico Sciacca, Op. cit., p. 261. 
El sentido de la libre voluntad es, por este reconocimiento, moral. En efecto, el reconocimiento voluntario es la sede de la libertad, y ésta se juega prioritariamente en el campo moral, en determinarse hacia el bien objetivo o subjetivo. Por ello es que la escuela rosminiana ha denominado a esta libertad como "bilateral". No se trata, entonces, de una mera abstracción metafísica, de una potencia meramente indiferente, o sea, que obre sin un motivo, sin una razón en este caso. Lo que indica es que el hombre está capacitado para hacer prevalecer uno de los motivos sobre los demás, de manera que se vuelva determinante para su obrar. En este sentido es que están vinculadas la libre voluntad y el libre actuar: el inicio de la acción consiste precisamente en la libre prevalencia de uno de los motivos que pueden tomarse como base para actuar. La acción, tomada externamente, es la obra que se lleva a cabo en el mundo real; pero esta acción tiene su fundamento en la libre determinación del motivo por el cual se obra ${ }^{18}$.

Esto presupone que las razones para obrar no determinan necesariamente a la voluntad, sino que ella, mediante la espontaneidad de su obrar, da a alguna de estas razones lo que necesita para volverse eficaz. En términos más exactos, la voluntad, al determinarse por una de las razones, al elegir una de ellas la vuelve un impulso mediante su libertad. Y esto es así porque las razones no son más que ideas, y las ideas no impulsan la realidad, en este caso al hombre mediante su voluntad; por tanto, es preciso que la razón prevaleciente se vuelva un impulso para que la obra se lleve a cabo. Y aquí está, a nuestro parecer, la libre voluntad en su determinación. El hombre, mediante este

\footnotetext{
${ }^{18}$ Antonio Rosmini, Antropologia in servizio della scienza morale, Op. cit., n. 606.
} 
ejercicio de la libre voluntad, se determina a sí mismo bien o mal moralmente, pues es capaz de hacer prevalecer una de las razones que tiene presentes para actuar. Y aquí está el fundamento del mérito o el demérito, de la virtud o el vicio, de la alabanza o el vituperio moral. El sentido de la libertad consiste en plegar la voluntad hacia el reconocimiento de los entes en su orden objetivo, ontológico; consiste en amarlos por aquello que son, no por aquello que el agente quisiera que fueran. El actuar externo tiene su sede aquí; la moralidad está, pues, en el reconocimiento del ente conocido; el sentido de la libertad está en amar libremente a los entes en su orden objetivo.

No sin razón dice Sciacca que "elegir (eleggere), empeñarse exponiéndose hasta el fondo, el máximo de responsabilidad de frente a nosotros mismos y a lo que elegimos: la libertad, inmersa en la iniciativa iluminada por el ser, retomada la luz sobre lo elegido, amado en su plenitud de valor, que sólo el amor hace conocer y apreciar en su profundidad inagotable" ${ }^{19}$. La libertad, vertida hacia el reconocimiento de los entes por lo que son, es la elección fundamental que perfecciona al hombre en cuanto tal, pues el hombre es esencialmente su voluntad. El hombre, mediante su voluntad, se vuelve bueno; y se vuelve bueno de manera absoluta, pues no hay nada nada bueno fuera de una buena voluntad: "Ni en el mundo, ni en general tampoco fuera del mundo, es posible pensar nada que pueda considerarse como bueno sin restricción a no ser tan sólo una buena voluntad" 20 .

\footnotetext{
${ }^{19}$ Michele Federico Sciacca, Op. cit., p. 272.

${ }^{20}$ Immanuel Kant, Fundamentación metafísica de las costumbres (Madrid, Emesa, 1977), Cap. I.

De ahí el dictum inmediato: "Der gute Wille hat seinen Wert in sich selbst".
} 


\section{Los límites de la libertad}

Pero la libertad, como hemos venido diciendo, y en lo que tiene toda la razón Hoyos, posee límites. No es, pues, una libertad absoluta, sino limitada y relativa. En primer lugar, la libertad puede limitarse por la carencia de elección. Puede faltar una razón suficiente que excite la elección, de manera que la voluntad modifique la marcha de sus operaciones voluntarias. Mientras el hombre está limitado a los solos bienes sensibles, no hay necesidad de elección, pues la sola espontaneidad de la voluntad, que sigue al instinto, es suficiente para decantarse hacia un bien y postergar otro. Incluso sucede esto cuando el sujeto se decanta por un bien sensible futuro; aquí todavía no entra en juego la libertad, pues aunque el bien futuro requiera del cálculo mental, no deja de ser un bien subjetivo. Elegir el bien mayor y postergar el menor no es otra cosa que seguir la espontaneidad de la voluntad; no es todavía la verdadera elección libre. Para que haya verdadera elección, acto precisamente de la libre voluntad, es necesario que el hombre visualice al bien objetivo. Sólo cuando se visualiza al bien objetivo, y cuando se "debe" obrar siguiéndolo, es que el hombre hace uso de su libre voluntad. Por ello con razón Rosmini afirma que los tratadistas morales hablan de la libre elección de la voluntad cuando hablan del obrar moral ${ }^{21}$. Igualmente, pueden faltar dos bienes contradictorios entre sí, esto es, uno subjetivo y otro objetivo, para que se dé la libre elección: la voluntad espontáneamente, faltando el bien objetivo, se determina por el subjetivo. Finalmente, ante la presencia de un bien

${ }^{21}$ Antonio Rosmini, Antropologia in servizio della scienza morale, Op. cit., n. 657. 
infinito, al menos hipotéticamente, la voluntad espontáneamente no puede sino seguirlo, en razón justamente de su infinitud; no hay dos contrarios que colisionen entre sí, por lo que la voluntad necesariamente sigue al bien infinito.

En segundo lugar, la libertad puede limitarse en cuanto viene a menos la fuerza práctica, es decir, cuando la fuerza de la elección disminuye porque le falta o bien fuerza a sí misma, o bien porque entra en conflicto con otras potencias, las cuales naturalmente se rebelan a los decretos de la voluntad, especialmente cuando se considera el imperium (en cuanto acto elícito, la voluntad sigue apeteciendo a pesar de no poder efectuar el imperium). Y esto sucede de múltiples maneras. Mencionemos al menos sucintamente algunas de ellas. En efecto, la eficacia de la fuerza práctica puede ser limitada por el instinto animal, junto con sus pasiones y hábitos, pues las dos son fuerzas activas que conviven en el hombre, y a veces el instinto limita a la libre voluntad cuando su fuerza es vehemente ${ }^{22}$. Puede ser limitada también por el

${ }^{22}$ Rosmini, a quien seguimos en esta clasificación de los límites de la voluntad, narra lo siguiente: "Du-Clos, en las memorias secretas de los reyes Luis XIV y Luis XV, narra que Enrique Julio de Borbón, hijo del gran Conde, se sentía a veces sumamente inclinado a actuar como perro, y en aquellos momentos comenzaba a ladrar con toda fuerza. Uno de estos accesos se dio un día en la recámara del rey Luis XIV. La presencia del monarca pudo contener un tanto la fuerza de tal instinto, pero no destruirlo. Por la fuerza fue llevado a la ventana, y sacando la cabeza hizo todo lo que pudo por sofocar el rumor del ladrido lo mejor que pudo, aunque realizó todos los otros actos de perro y los movimientos de la boca. ¿`No es evidente que, de poder la voluntad vencer tales deseos del instinto, lo habría hecho en tal momento?”, Antropologia in servizio della scienza morale, n. $676,1^{\circ}$. Ahora bien, que el instinto doblegue a la fuerza práctica de la voluntad se da de dos modos, o bien porque la voluntad, aun queriendo 
instinto humano, acompañado de pasiones, reminiscencias, asociaciones, hábitos, etcétera. $\mathrm{El}$ instinto humano es la tendencia a la propia grandeza, a la sociedad, a la gloria, a la ciencia, a la simpatía, etcétera, que a veces pueden entran en conflicto o colisión con la voluntad, que es la que debe obrar siguiendo la ley moral ${ }^{23}$.

con acto elícito un objeto, está impedida para efectuar el imperium; o bien puede el instinto eliminar de tal manera a la libre voluntad, que el actuar del hombre se vuelve ciego en este sentido.

${ }^{23} \mathrm{E} 1$ siguiente pasaje resulta de gran importancia para comprender qué es el instinto humano: "El instinto bumano es más elevado y alto que el instinto meramente animal. Pero toma los materiales para sus operaciones en gran parte de la misma animalidad. $\mathrm{El}$ amor, por ejemplo, tiene su parte material en la inclinación física de los dos sexos; a la simpatía vienen suministrados los materiales en buena parte por la imaginación; la amistad confina a veces con el amor, y toma tal coloración que la vuelve más viva y animada, pues vive también de reminiscencias e imágenes; la sociabilidad trae su ser por la semejanza de la naturaleza humana, y de las necesidades intercambiables, cosas todas que por los sentidos nos vienen indicadas. El alimento de la gloria es el sonido de la voz, por lo que suele tomarse de la fama sonante y de la tromba; y así puede decirse de todas las otras inclinaciones subjetivas del hombre, que se recogen todas juntas tomadas bajo la denominación del instinto humano. Empero, todo lo que suministra la animalidad es sólo la materia. La forma del instinto humano es colocada por la razón. Esta última elabora de manera admirable la materia que le suministran los sentidos; la sublima, transforma, amplifica, espiritualiza, diviniza. Por tanto, es una cosa esencialmente diversa este instinto humano, que no tiene confines porque no los tiene la inteligencia, y el instinto animal", Antonio Rosmini, Antropologia in servizio della scienza morale, n. 684. 
En tercer lugar, la libre voluntad puede verse limitada por el juicio, en cuanto éste es el inicio de la volición, es decir, del acto elícito. En efecto, la volición se apoya sobre un juicio, un juicio por el cual el hombre se declara a sí mismo que un cierto partido es mejor y preferible a su contrario. Este juicio es el nodo que une la volición con la elección; es el objeto inmediato de la elección misma. En otras palabras, el hombre delibera, elige efectuar un juicio más que el otro. Con este juicio tiene inicio la volición. El juicio es el término próximo de la elección y el principio de la volición. Por ello, si el juicio es limitado, también se limita el actuar de la voluntad y, en consecuencia, la libre voluntad ${ }^{24}$. Así pues, si hay limitaciones, en primer lugar, en torno al juicio teórico sobre la cosa, como cuando no se logra comprender bien a bien qué es, es evidente que lo que pueda hacerse libremente con ella está limitado por nuestra carencia de conocimiento (el caso extremo se da cuando el sujeto es incapaz de distinguir qué es lo que percibe). Además, puede suceder que el juicio práctico, por su lado, sea falseado por el ímpetu de los impulsos, que llevan a concluir la deliberación con un juicio que no sea el idóneo, especialmente en términos normativos. Aquí entran también los límites que establecen las opiniones ya formadas. Efectivamente, la opinión es una sentencia que recibe un individuo y que no somete a examen, pues la estima verdadera. Pero sucede a menudo que esta sentencia es falsa, y resulta más perniciosa si se trata de una sentencia de índole moral, pues, como dijimos, el sentido de la libertad se halla en su perfeccionamiento moral, ante lo cual tiene la máxima responsabilidad.

${ }^{24}$ Cf. Antonio Rosmini, Antropologia in servizio della scienza morale, Op. cit., n. 704. 
Por último, también podría argüirse como límite de la libre voluntad las voliciones virtuales y habituales. Las voliciones virtuales son las disposiciones de la voluntad que, ante un cierto objeto, aunque no lo conozca bien a bien, indudablemente lo quiere, a pesar de que ignore su inclinación o bien a lo que es conducida. Por su parte, la volición habitual es la disposición de la voluntad que no está solamente inclinada a querer dicho objeto, sino que está determinada a quererlo. En cierta manera, ambas determinan la libertad en su obrar, porque la influyen de tal manera que la inclinan casi irremediablemente a sus objetos. Es lo que sucede con la ceguera a los valores morales a la que Von Hildebrand se refiere de continuo. Nos habituamos de tal manera a despreciar ciertos objetos, como puede ser la virtud, y a determinarnos por otros de menor valor, que la voluntad, para evitar la fatiga de tener que deliberar y elegir, se determina de inmediato a través de su espontaneidad. Pero en todos estos casos "la ceguera moral se halla más bien fundada en el ser moral, en la posición y la actitud fundamental de la persona, y es por ello siempre culpable en cierto sentido" ${ }^{25}$. El hombre, al habituarse a un cierto género de objetos, puede cegarse a otros que resultarían de mayor valía moral. Por ello es que hay culpabilidad, pues la libertad se va limitando cada vez más en su ejercicio y es más difícil escapar a tal estado.

${ }^{25}$ Cf. Dietrich von Hildebrand, Moralidad y conocimiento ético de los valores (Madrid, Cristiandad, 2006), p. 45. 


\section{Condusiones}

En este trabajo nos propusimos sostener que el sentido de la libertad consiste en la moralidad, pues de manera radical se manifiesta en el reconocimiento del ser de los entes en su orden. En otros términos, reconocer lo que se conoce es la raíz de la moralidad, y la libertad, si es que pretende desarrollarse para alcanzar su punto culminante, se debe dirigir hacia tal sendero. Revisamos, primero, la noción de voluntad, pues nos parece imprescinimprescindible aclarar en qué consiste para situar ahí a la libertad, pues con justicia se habla de libre voluntad. Ahí discutimos con Hoyos, quien sostiene la falta de interés filosófico por tal noción, decantándose por la noción de libertad de acción. Pero demostramos que es más fundamental la libertad de la voluntad, pues el liberum arbitrium consiste más bien en un juicio que efectuamos sobre la bondad de la cosa conocida, a partir del cual actuamos. De ahí brota la necesidad normativa de la libertad, que para desarrollarse adecuadamente ha de hacerlo desde una perspectiva moral. Mas también hemos visto algunos de los límites a los que subyace, y que menguan e incluso, en algunos casos, impiden el libre ejercicio.

\section{Bibliografia}

Aquinatis, Thomae, Summa theologiae, Petri Fiaccadori, Parma, 1852.

Aristotelis, Ethica Nicomachea (edición de Franciscus Susemihl), Teubneri, Lipsae, 1903, 280pp.

Buganza, Jacob, El ser y el bien, Edizioni Rosminiane Sodalitas, Stresa, 2010, 116pp. 
Sobre el sentido moral de la libertad

Buganza, Jacob, Filosofía de la mente, voĩ y libertad, Universidad Veracruzana, Xalapa, 2014, 164pp.

De Caro, Mario, Il libero arbitrio. Una introduzione, Laterza, Roma y Bari, 2009 (3a. ed), 192pp.

Hildebrand, Dietrich von, Moralidad y conocimiento ético de los valores (traducción de Juan Miguel Palacios), Cristiandad, Madrid, 2006, 217pp.

Hoyos, Luis Eduardo, “El sentido de la libertad”, en: Ideas y valores, n. 141, Bogotá, 2009, pp. 85-107.

Kant, Immanuel, Fundamentación metafisica de las costumbres, Emesa, Madrid, 1977, 188pp.

Marion, Jean-Luc, Cours sur la volonté, Presses Universitaires de Louvain, Louvain, 2014, 208pp.

Morando, Giuseppe, Il problema del libero arbitrio, Cogliati, Milano, 1895, 351pp.

Rosmini, Antonio, Psicologia, t. II, Città Nuova, Roma, 1988, 373pp.

Rosmini, Antonio, Antropologia in servizio della scienza morale, Città Nuova, Roma, 1981, 590pp.

Rosmini, Antonio, Principi della scienza morale, Città Nuova, Roma, 1990, 505pp.

Sciacca, Michele Federico, La libertà e il tempo, Marzorati, Milano, 1965, 343pp.

Dr. Jacob Buganza

Instituto de Filosofía

Universidad Veracruzana Tuxpan No. 29 Fracc. Veracruz

Xalapa, Veracruz. C. P. 91020

Tel. (228) 8154285 
